\title{
Measures of noncompactness on $w$-distance spaces
}

\author{
Aleksandar Kostić*
}

\begin{abstract}
The aim of this paper is to provide a new framework for the study of measures of noncompactness in generalized metric spaces. Firstly, we introduce the notion of $w$-measure of noncompactness on metric spaces with a $w$-distance and extend the diameter and Kuratowski functionals to this setting. At the end we give a characterization of metric completeness via our main results, providing a new answer to the open question mentioned by Arandjelović in his $\mathrm{PhD}$ thesis [2].
\end{abstract}

\section{INTRODUCTION AND PRELIMINARIES}

The notion of measure of noncompactness seems to have been introduced by Kuratowski [10] in 1930 to study some generalizations of the famous Cantor intersection theorem. However, it was not until some decades later that it was applied in a much wider context of functional analysis, first and most notably by Darbo [4] in fixed point theory. For more on measures of noncompactness and applications we refer to the classical book [3], as well as $[11,12]$. We begin with the definition of total boundedness, for the readers' convenience (see e.g. [11, 12]).

Definition 1. A set $A$ in a metric space $(X, d)$ is said to be totally bounded if for every $\varepsilon>0$ it can be covered by a finite number of open balls of radius $\varepsilon$.

The following is the definition of Kuratowski measure of noncompactness $([3,10,11,12])$.

Definition 2. Let $(X, d)$ be a metric space. Kuratowski measure of noncompactness of a bounded set $A \subseteq X$, denoted by $\alpha(A)$, is the infimum of all $\varepsilon>0$ such that $A$ can be covered by a finite number of sets whose diameter is less than $\varepsilon$.

2020 Mathematics Subject Classification. Primary: 47H08; Secondary: 54E50.

Key words and phrases. Measures of noncompactness, metric spaces, w-distance.

Full paper. Received 13 May 2020, revised 2 October 2020, accepted 30 November 2020, available online 16 March 2021.

*This research has been supported by the Ministry of Science, Education and Technological Development of the Republic of Serbia through the Faculty of Sciences and Mathematics in Niš, Contract No. 451-03-68/2020-14/200124. 
Theorem 1 (Kuratowski [10]). Let $(X, d)$ be a complete metric space, and let $\left\{F_{n}\right\}$ be a sequence of nonempty closed and bounded subsets of $X$ such that $F_{n+1} \subseteq F_{n}$ for all $n \in \mathbb{N}$ and $\lim _{n \rightarrow \infty} \alpha\left(F_{n}\right)=0$. Then, $\cap_{n=1}^{\infty} F_{n}$ is a nonempty compact subset of $X$.

In 1996 Kada et al. [6] introduced and studied the notion of $w$-distance in a metric space in order to generalize several important fixed point theorems. Its applications in best proximity point theory have been extensively studied by the author of the present paper (independently or in co-authorship) in $[7,8,9]$.

Definition 3. Let $(X, d)$ be a metric space, and let $p: X \times X \rightarrow[0, \infty)$ be a function. Then $p$ is called a $w$-distance on $X$ if it satisfies the following conditions:

(1) $p(x, y) \leq p(x, z)+p(z, y)$ for all $x, y, z \in X$;

(2) $p(x, \cdot): X \rightarrow[0, \infty)$ is a lower semicontinuous function for all $x \in X$;

(3) for all $\varepsilon>0$ there exists $\delta>0$ such that $p(z, x) \leq \delta$ and $p(z, y) \leq \delta$ imply $d(x, y) \leq \varepsilon$.

Recall that a function $f$ defined on a metric space $X$ is said to be lower semicontinuous at a point $x \in X$ if either $\liminf _{x_{n} \rightarrow x} f(x)=\infty$ or $f(x) \leq$ $\liminf _{x_{n} \rightarrow x} f\left(x_{n}\right)$ for any sequence $\left\{x_{n}\right\} \subseteq X$ converging to $x$.

Very recently, Kostić et al. [9] used a $w$-distance such that $p(\cdot, y)$ is also lower semicontinuous for all $y \in X$, which they called the $w_{0}$-distance. In this paper every $w$-distance is assumed to be a $w_{0}$-distance if not specified otherwise.

Some important properties of $w$-distances are given in the next lemma.

Lemma $1([6])$. Let $(X, d)$ be a metric space with a $w$-distance $p$. Then we have that:

(i) $p(x, y)=0$ and $p(x, z)=0$ imply $y=z$, for all $x, y, z \in X$;

(ii) if $\left\{\alpha_{n}\right\} \subseteq[0, \infty)$ converges to 0 and $p\left(x_{n}, x_{m}\right) \leq \alpha_{n}$, for all $m, n \in \mathbb{N}$ with $m>n$, then $\left\{x_{n}\right\}$ is a Cauchy sequence in $X$.

In this paper, we introduce the notion of $w$-measure of nocnompactness on a metric space with a $w$-distance. To this end, we shall use the axiomatic approach similar to that introduced by Arandjelović in $[1,2]$. Then we generalize the notions of diameter and Kuratowski measure of noncompactness in this setting, and show that they are indeed $w$-measures of noncompactness. We also provide a new characterization of metric completeness.

\section{MAIN RESUlts}

As already mentioned, we shall modify the axiomatic definition of measures of noncompactness on metric spaces used by Arandjelović [1, 2] in order to extend this notion to metric spaces with a $w$-distance. If $(X, d)$ is 
a metric space and $A$ its subset, we shall denote the partitive set of $X$ by $P(X)$ and the closure and diameter of $A$ by $\bar{A}$ and $\delta(A)$ respectively.

Definition 4. Let $(X, d)$ be a metric space with a $w$-distance $p$, and let $A$ be its subset. The $p$-diameter of the set $A$, denoted by $\delta_{p}(A)$, is defined by

$$
\delta_{p}(A)=\sup _{x, y \in A} p(x, y),
$$

(see [5]).

If the above supremum exists and is finite, we say that the set $A$ is $p$ bounded; otherwise, $A$ is $p$-unbounded.

Definition 5. Let $(X, d)$ be a metric space with a $w$-distance $p$. The function $\phi: P(X) \rightarrow[0, \infty)$ is said to be $w$-measure of noncompactness if it satisfies the following conditions:

(1) $\phi(A)=\infty$ if and only if $A$ is $p$-unbounded;

(2) $\phi(A)=\phi(\bar{A})$;

(3) if $\phi(A)=0$, then $A$ is totally bounded;

(4) if $A \subseteq B$, then $\phi(A) \leq \phi(B)$;

(5) if $X$ is complete, and $\left\{F_{n}\right\}$ is a sequence of nonempty closed subsets of $X$ such that $F_{n+1} \subseteq F_{n}$ for all $n \in \mathbb{N}$ and $\lim _{n \rightarrow \infty} \phi\left(F_{n}\right)=0$, then $\cap_{n=1}^{\infty} F_{n}$ is a nonempty compact subset of $X$.

Now we introduce the first $w$-measure of noncompactness.

Theorem 2. If $(X, d)$ is a metric space with a $w$-distance $p$, then $\delta_{p}$ is a $w$-measure of noncompactness on $X$.

Proof. It suffices to show that the function $\delta_{p}$ satisfies all conditions in the Definition 5 .

(1) This is obvious by the Definition 4 .

(2) Since $A \subseteq \bar{A}$, it is obvious by the Definition 4 that $\delta_{p}(A) \leq \delta_{p}(\bar{A})$, so we need to prove the converse inequality. Suppose, to the contrary, that $\delta_{p}(A)<\delta_{p}(\bar{A})$. Then there exist $x, y \in \bar{A}$ such that $\delta_{p}(A)<$ $p(x, y)$. Since $x, y \in \bar{A}$, there exist two sequences $\left\{x_{n}\right\},\left\{y_{n}\right\}$ in $A$ converging to $x$ and $y$ respectively. But then using the lower semicontinuity of $p$ with respect to both variables we get:

$$
\begin{aligned}
& \delta_{p}(A)<p(x, y) \leq \liminf _{n \rightarrow \infty} p\left(x_{n}, y\right) \leq \\
& \leq \liminf _{n \rightarrow \infty} \liminf _{m \rightarrow \infty} p\left(x_{n}, y_{m}\right) \leq \delta_{p}(A),
\end{aligned}
$$

which is a contradiction.

(3) Suppose that $A$ is a subset of $X$ such that $\delta_{p}(A)=0$. By the Definition 4 , that means that $p(x, y)=0$ for all $x, y \in A$. Assume that $A$ contains two distinct points $x$ and $y$. Then we have $p(x, y)=$ 0 and $p(y, x)=0$, which implies that $p(x, x) \leq p(x, y)+p(y, x)=0$, i.e. $p(x, x)=0$. But now, from $p(x, x)=0$ and $p(x, y)=0$, by 
Lemma 1 (i) we obtain $x=y$, a contradiction. Hence, $A$ does not contain more than one point, i.e. $A$ is either an empty set or a singleton. In both cases, $A$ is totally bounded.

(4) Evident, again by Definition 4.

(5) Let $\left\{F_{n}\right\}$ be a sequence of subsets as in (5) of Definition 5, and let $F$ be its intersection. Since $F \subseteq F_{n}$ for all $n \in \mathbb{N}$, by (4) we have $\delta_{p}(F) \leq \delta_{p}\left(F_{n}\right) \rightarrow 0$. Thus, $\delta_{p}(F)=0$, which by the proof of $(3)$ implies that $F$ is either empty or a singleton set. Let us choose a point $x_{n} \in F_{n}$ for all $n \in \mathbb{N}$. We shall prove that the sequence $\left\{x_{n}\right\}$ converges to a point $x \in F$, so it must be $F=\{x\}$. Indeed, for all $m, n \in \mathbb{N}$ with $m>n$ we have $p\left(x_{n}, x_{m}\right) \leq \delta_{p}\left(F_{n}\right) \rightarrow 0$ as $n \rightarrow \infty$, which by Lemma 1 (ii) implies that $\left\{x_{n}\right\}$ is a Cauchy sequence in $X$, so it converges to a point $x \in X$ because $X$ is complete. It is easy to see that $x \in F_{n}$ for all $n \in \mathbb{N}$, and therefore $x \in F$. Hence, $F=\{x\}$, which is of course nonempty and compact.

Now we can introduce the Kuratowski $p$-measure of noncompactness.

Definition 6. Let $(X, d)$ be a metric space with a $w$-distance $p$, and let $A$ be its subset. Then we define the Kuratowski p-measure of noncompactness of the set $A$, denoted by $\alpha_{p}(A)$, as the infimum of all $\varepsilon>0$ such that $A$ can be covered by a finite number of subsets in $X$ whose $p$-diameter is less than $\varepsilon$.

Theorem 3. If $(X, d)$ is a metric space with a w-distance $p$, then $\alpha_{p}$ is a $w$-measure of noncompactness on $X$.

Proof. Again, we shall show that $\alpha_{p}$ satisfies all conditions of Definition 5.

(1) This follows trivially from the Definition 6 .

(2) Since $A \subseteq \bar{A}$ it is evidently $\alpha_{p}(A) \leq \alpha_{p}(\bar{A})$ by Definition 6. To prove the converse inequality, let $\varepsilon>0$ be arbitrary, and let $S_{i}$, $i=1, \ldots, n$ be subsets of $X$ such that $\delta_{p}\left(S_{i}\right)<\varepsilon$ for all $i=1, \ldots, n$ and $A \subseteq \cup_{i=1}^{n} S_{i}$. Then we have $\bar{A} \subseteq \overline{\cup_{i=1}^{n} S_{i}}=\cup_{i=1}^{n} \bar{S}_{i}$. Since $\delta_{p}\left(S_{i}\right)=\delta_{p}\left(\bar{S}_{i}\right)$, we get $\alpha_{p}(\bar{A}) \leq \alpha_{p}(A)$.

(3) Let $A$ be a subset of $X$ such that $\alpha_{p}(A)=0$. Fix an arbitrary $\varepsilon>0$, and choose $\delta=\delta\left(\frac{\varepsilon}{2}\right)$ corresponding to $\frac{\varepsilon}{2}$ in Definition 3 (3). Let $A_{j}$ be any element of the covering of $A$ corresponding to $\delta$ in Definition 6 , so $\delta_{p}\left(A_{j}\right)<\delta$. It then follows that for every $x, y \in A_{j}$ we have $p(x, x)<\delta$ and $p(x, y)<\delta$. But this in turn implies $d(x, y) \leq \frac{\varepsilon}{2}<\varepsilon$ by Definition 3 (3), which means that $\delta\left(A_{j}\right)<\varepsilon$, i.e. diameter of every component in the covering of $A$ is also less than $\varepsilon$. Since $\varepsilon>0$ was arbitrary, we conclude that the set $A$ is totally bounded (see Definition 1).

(4) Obvious by Definition 6 .

(5) Let $\left\{F_{n}\right\}$ be the sequence as in Definition 5 (5), and let $F$ be its intersection. Then $F$ is closed because every $F_{n}$ is closed. Since 
$F \subseteq F_{n}$ for any $n \in \mathbb{N}$, we have $\alpha_{p}(F) \leq \alpha_{p}\left(F_{n}\right) \rightarrow 0$ when $n \rightarrow \infty$. So, $\alpha_{p}(F)=0$, which implies that $F$ is a totally bounded set - this has been proven under (3). But $F$ is complete, so $F$ is actually relatively compact. It is also closed, which means it is compact. That $F$ is nonempty can be shown in the same way as in the proof of Kuratowski's theorem (Theorem 1), see e.g. [3, 10, 11].

\section{Characterizations of metric Completeness}

It is well known that Cantor intersection theorem characterizes metric completeness. We note that similar results have recently been obtained in the setting of the so-called partial metric spaces in [13].

However, the problem of characterizing metric completeness via measures of noncompactness has long been open, see [2, Section I.5].

It is also known that Suzuki and Takahashi [14] characterized the metric completeness via generalized Banach's fixed point theorem on metric spaces with a $w$-distance.

Motivated by these results, we find it natural to ask if metric completeness can be characterized using $w$-measures of noncompactness introduced in the present paper. We shall give a positive answer in the next theorem.

Theorem 4. Let $(X, d)$ be a metric space with a w-distance $p$. The following conditions are equivalent:

(i) $X$ is complete;

(ii) Every sequence $\left\{F_{n}\right\}$ of nonempty closed subsets in $X$ such that $F_{n+1} \subseteq F_{n}$ for all $n \in \mathbb{N}$ and $\lim _{n \rightarrow \infty} \delta_{p}\left(F_{n}\right)=0$ has a singleton intersection;

(iii) Every sequence $\left\{F_{n}\right\}$ of nonempty closed subsets in $X$ such that $F_{n+1} \subseteq F_{n}$ for all $n \in \mathbb{N}$ and $\lim _{n \rightarrow \infty} \alpha_{p}\left(F_{n}\right)=0$ has a nonempty compact intersection.

Proof. We shall prove the following chain of implications.

(i) $\Rightarrow$ (iii):

This is shown in part (5) of the proof of Theorem 3.

(iii) $\Rightarrow$ (ii):

Suppose that the sequence $\left\{F_{n}\right\}$ satisfies the conditions under (ii). Since by Definition $6, \alpha_{p}\left(F_{n}\right) \leq \delta_{p}\left(F_{n}\right)$ for all $n \in \mathbb{N}$, it is easily seen that the sequence $\left\{F_{n}\right\}$ also satisfies the conditions under (iii), so its intersection $F$ is a nonempty compact subset of $X$. Also, $\delta_{p}(F)=0$, which is only possible if $F$ is a singleton (see the proof of Theorem 3).

(ii) $\Rightarrow$ (i):

Suppose that (ii) holds and $X$ is not complete. Then there exists a Cauchy sequence $\left\{x_{n}\right\}$ in $X$ which is not convergent. It can be easily shown that the set $F=\left\{x_{n}: n \in \mathbb{N}\right\}$ is bounded and closed. Let $p: X \times X \rightarrow[0, \infty)$ 
be a $w$-distance on $X$ defined by

$$
p(x, y)= \begin{cases}d(x, y), & \text { if } x, y \in F, \\ 2 \delta(F), & \text { otherwise }\end{cases}
$$

(see [6, Example 7]). Let $F_{n}=\left\{x_{k}: k \geq n\right\}$ for all $n \in \mathbb{N}$. Then we have that every $F_{n}$ is a nonempty closed subset of $X$ such that $F_{n+1} \subseteq F_{n}$ for all $n \in \mathbb{N}$, and

$$
\lim _{n \rightarrow \infty} \delta_{p}\left(F_{n}\right)=\lim _{n \rightarrow \infty} \sup _{m \geq n} p\left(x_{n}, x_{m}\right)=\lim _{n \rightarrow \infty} \sup _{m \geq n} d\left(x_{n}, x_{m}\right)=0,
$$

since $\left\{x_{n}\right\}$ is Cauchy. But then by (ii) we obtain that $\cap_{n=1}^{\infty} F_{n}=\{x\}$ for some $x \in X$, which is impossible, because $\left\{x_{n}\right\}$ would then converge to $x$.

\section{Conclusion}

In this paper we have introduced the notions of $w$-measures of noncompactness on metric spaces with a $w$-distance. Our main results generalize the well known Cantor intersection theorem and Kuratowski's theorem [10]. The main results have also been employed to characterize metric completeness, giving a new answer to the open problem from [2].

We strongly believe that the results of the present paper can be applied to various problems of nonlinear analysis, such as in fixed point theory and theory of nonlinear integral and differential equations. Moreover, our results could be extended further in the setting of various other generalized metric structures, as well as uniform spaces.

\section{REFERENCES}

[1] I. D. Arandjelović, Measures of noncompactness on uniform spaces, Mathematica Moravica, 2 (1998), 1-8.

[2] I. Arandjelović, Stavovi o presecanju i njihove primene u nelinearnoj analizi (in Serbian), PhD thesis, Faculty of Mathematics, University of Belgrade, 1999.

[3] J. Banaś, M. Mursaleen, Sequence spaces and measures of noncompactness with applications to differential and integral equations, Springer, 2014.

[4] G. Darbo, Punti uniti in trasformazioni a codominio non compatto, Rendiconti del Seminario Matematico della Università di Padova, 24 (1955), 84-92.

[5] D. Ilić, V. Rakočević, Common fixed points for maps on metric space with w-distance, Applied Mathematics and Computation, 199 (2008), 599-610.

[6] O. Kada, T. Suzuki, W. Takahashi, Nonconvex minimization theorems and fixed point theorems in complete metric spaces, Mathematica Japonica, 44 (1996), 381-391.

[7] A. Kostić, Best proximity points revisited, Filomat, 33 (16) (2019), 5159-5166. 
[8] A. Kostić, E. Karapinar, V. Rakočević, Best proximity points and fixed points with $R$ functions in the framework of $w$-distances, Bulletin of the Australian Mathematical Society, 99 (3) (2019), 497-507.

[9] A. Kostić, V. Rakočević, S. Radenović, Best proximity points involving simulation functions with $w_{0}$-distances, Revista de la Real Academia de Ciencias Exactas, Físicas y Naturales. Serie A. Matemáticas, 113 (2019), 715-727.

[10] K. Kuratowski, Sur les espaces complets, Fundamenta Mathematicae, 15 (1930), 301-309.

[11] E. Malkowsky, V. Rakočević, Introduction into the theory of sequence spaces and measures of noncompactness, Zbornik Radova, 17 (2000), 143-234.

[12] V. Rakočević, Measures of noncompactness and some applications, Filomat, 12 (2) (1998), 87-120.

[13] M. Singha, K. Sarkar, Towards Cantor intersection theorem and Baire category theorem in partial metric spaces, Matematički Vesnik, 69 (2) (2017), 126-132.

[14] T. Suzuki, W. Takahashi, Fixed point theorems and characterizations of metric completeness, Topological Methods in Nonlinear Analysis, 8 (1996), 371-382.

\author{
Aleksandar Kostić \\ UNIVERSITY OF Niš \\ Faculty of Sciences and Mathematics \\ VIŠEGRADSKA 33 \\ 18000 NiS̆ \\ SERBIA \\ E-mail address: akos2804@gmail.com
}

